\title{
Desarrollo de una Escala de Conducta Prosocial para Adultos en México
}

\author{
Development of a Prosocial Behavior Scale for Adults in Mexico
}

\author{
Marco Antonio De La Cruz Perez ${ }^{1}$, Sofía Rivera Aragón ${ }^{2}$, Adrián Medina Liberty ${ }^{3}$, \\ Tonatiuh García Campos ${ }^{4}$, Rolando Díaz Loving ${ }^{5}$ y Angelica Romero Palencia ${ }^{6}$
}

\begin{abstract}
Resumen
La evaluación de la conducta prosocial está dirigida principalmente hacia niños y adolescentes. Aquellas escalas enfocadas en adultos son dirigidas a estudiantes o evalúan motivaciones y situaciones subyacentes. Por lo tanto, el propósito del presente estudio fue desarrollar una escala de autoinforme para evaluar la conducta prosocial en adultos y obtener sus propiedades psicométricas (dimensiones, validez predictiva y confiabilidad). Participaron 761 personas de 18 a 75 años $(M=29.22, D E=12.51)$. La escala quedó compuesta por 22 reactivos distribuidos en 4 factores, con un alfa de Cronbach de .89 y $45.8 \%$ de varianza explicada. Los resultados muestran que la escala es adecuada para evaluar de manera valida y confiable la conducta prosocial en adultos de población abierta de la ciudad de México.
\end{abstract}

Palabras clave: altruismo, empatía, positivo, prosocialidad, voluntariado

\begin{abstract}
The assessment of prosocial behavior is primarily geared towards children and adolescents. The scales focusing on adults target students or assess underlying motivations and situations. Therefore, the aim of this study was to develop a self-report scale to assess prosocial behavior in adults and obtain its psychometric properties. (dimensions, predictive validity, and reliability). 761 individual aged between 18 and 75 years old participated $(\mathrm{M}=29.22, \mathrm{SD}=12.51)$. The scale consisted of 22 items distributed across 4 factors, with a Cronbach's alpha of .89 and $45.8 \%$ of variance accounted for. The results show that the scale is adequate to evaluate the prosocial behavior in adults of the open population of Mexico City in a valid and reliable manner.
\end{abstract}

Keywords: altruism, empathy, positive, prosociality, volunteerism

La presente investigación fue realizada gracias al apoyo otorgado por el Consejo Nacional de Ciencia y Tecnología (CONACyT) a través de la Beca Nacional al primer autor de este artículo (CVU: 927238).

\footnotetext{
${ }^{1}$ Lic. en Psicología. Facultad de Psicología, Universidad Nacional Autónoma de México. Doctorante inscrito en el programa de maestría y doctorado de la facultad de psicología, UNAM. Av. Universidad 3004 cub. D008 Edificio D Mezzanine, Facultad de Psicología, UNAM 04510, Ciudad de México, México. Tel.: 7717123440. Correo: marcod_cruz@outlook.com

${ }^{2}$ Dra. en Psicología Social. Facultad de Psicología, Universidad Nacional Autónoma de México. Profesor titular "C" TC de la Facultad de Psicología UNAM. Av. Universidad 3004 cub. D008 Edificio D Mezzanine, Facultad de Psicología, UNAM 04510, Ciudad de México, México. Tel.: 525556222259. Correo: sofiar@unam.mx

${ }^{3}$ Dr. en Antropología Cultural. Facultad de Psicología, Universidad Nacional Autónoma de México. Profesor titular "B" TC de la Facultad de Psicología UNAM. Av. Universidad 3004, Facultad de Psicología, UNAM 04510, Ciudad de México, México. Tel.: 5555231824. Correo: amedina@unam.mx

${ }^{4}$ Dr. en Psicología. Universidad de Guanajuato. Profesor titular "A" TC de la Universidad de Guanajuato. Blvd Puente Milenio 1001, fracción del predio San Carlos, Leon, Gto, CP 3670, México. Tel.: 4772674900 ext. 3643. Correo: tonat99@ hotmail.com

${ }^{5}$ Dr. en Psicología Social. Facultad de Psicología, Universidad Nacional Autónoma de México. Profesor titular "C" TC, Def. de la Facultad de Psicología UNAM. Av. Universidad 3004 cub. D009 Edificio D Mezzanine, Facultad de Psicología, UNAM 04510, Ciudad de México, México. Tel.: 5536431735. Correo: rdiazl@unam.mx

${ }^{6}$ Dra. en Psicología. Facultad de Ciencias Humana, Universidad La Salle Pachuca. Profesora de asignatura. Río tamesis 106 col. ISSSTE cp. 42083 Pachuca Hidalgo, México. Tel.: 7715668996. Correo: aromero@lasallep.mx
}

Revista Iberoamericana de Diagnóstico y Evaluación - e Avaliação Psicológica. RIDEP · No61 · Vol.4 · 65-79 · 2021

ISSN: 1135-3848 print /2183-6051online 


\section{Introducción}

La importancia de estudiar la conducta prosocial radica en que promueve el bienestar personal (Aknin, Whillans, Norton, \& Dunn, 2019) y el establecimiento de relaciones interpersonales positivas (Palomar \& Victorio, 2018). De igual forma, es fundamental para la protección de la salud, para el apoyo en situaciones de desastres y el combate de la desigualdad (Grant \& Dutton, 2012). Debido a las implicaciones positivas de la conducta prosocial, es importante conocer los aspectos que subyacen su expresión. Por ello es necesario contar con mediciones precisas del constructo (Eisenberg \& Spinrad, 2014).

Los instrumentos de evaluación de conducta prosocial son muy diversos en los elementos que contemplan, además de conductas, algunos incorporan motivaciones (Auné, Abal, \& Attoresi, 2019) y situaciones subyacentes (Carlo \& Randall, 2002). Si bien la motivación (Gebauer, Riketta, Broemer, \& Maio, 2008; Mujcic \& Leibbrandt, 2017; Sheldon, Wineland, Venhoeven, \& Osin, 2016) y situación (LaningaWijnen, Harakeh, Dijkstra, Veenstra, \& Vollebergh, 2018; Lapo \& Bustamante, 2018; Moradi, Van Quaquebeke, \& Hunter, 2018) son aspectos estrechamente relacionados con el constructo, incorporarlos en un solo instrumento representa un gran desafío debido a lo numeroso de cada elemento y a la gran cantidad de posibles combinaciones. Ante esto, algunos autores recomiendan aproximarse de forma independiente a cada aspecto (Bravo \& Reyes-Lagunes, 2009; Eisenberg \& Spinrad, 2014; Gebauer et al., 2008; McGuire, 1994, Mesurado, 2014), por lo que, para este estudio serán consideradas únicamente las conductas.

La conducta prosocial es todo comportamiento voluntario realizado para beneficiar a otros (Dovidio, Piliavin, Schroeder, \& Penner, 2006; Eisenberg, Spinrad, \& Knafo, 2015). Si bien refiere a la promoción del bienestar ajeno (Grusec, Hastings, \& Almas, 2011), la definición no abarca qué acciones son prosociales. Debido a la falta de especificidad, Gebauer et al. (2008) recomiendan delimitar los comportamientos prosociales particulares de cada estudio, para que los resultados y conclusiones derivados sean más específicos.
Algunos autores han categorizado a la conducta prosocial en comportamientos de ayuda y voluntariado. La ayuda es cualquier comportamiento de auxilio o asistencia hacia otros (Collet \& Morrisey, 2007). McGuire (1994) especifica cuatro tipos: Ayuda casual (e. g. sujetar o levantar objetos, etc.), ayuda personal sustancial (e. g. dar o prestar objetos valiosos), ayuda emocional (e. g. compañía, consuelo, escucha) y ayuda de emergencia (e. g. asistir a un herido). Con relación al voluntariado, es un comportamiento planificado, realizado frecuentemente e institucionalizado (Penner, 2002). Algunos ejemplos son el apoyo físico o material hacia grupos vulnerables, instituciones educativas, religiosas y comunitarias (Collet \& Morrisey, 2007).

Otra aproximación conceptual, realizada por De La Cruz y Rivera-Aragón (en prensa), exploró el significado psicológico y la conceptuación de la conducta prosocial en adultos de la ciudad de México. A través de redes semánticas y preguntas abiertas, los resultados mostraron que la conducta prosocial está compuesta por acciones como ayudar, apoyar, compartir, dar, escuchar, entre otras; así mismo, comprende comportamientos más específicos como cuidar las áreas verdes, ceder el asiento, dar comida a quien lo necesita, hacer donaciones, realizar voluntariados, cargar objetos pesados, etc. Con base en estos hallazgos, se delimitaron las conductas prosociales para la presente investigación. Esta exploración en el contexto mexicano resulta relevante puesto que, lo que es considerado prosocial está social y culturalmente determinado (House et al., 2020; Penner, Dovidio, Piliavin, \& Schroeder, 2005).

Con respecto al por qué la gente es prosocial, distintas teorías responden la cuestión con aproximaciones biológico-evolutivas, cognitivoafectivas y psicosociales. Desde la perspectiva biológica, genes implicados en la actividad de la dopamina, serotonina, oxitocina y vasopresina se asocian con la conducta prosocial (Fortuna \& Knafo, 2014). Similarmente, la perspectiva evolutiva propone que existen tendencias innatas prosociales, cuyo fin es propiciar la supervivencia de la especie (Amici, 2015; Warneken \& Tomasello, 2009). Ambas perspectivas consideran que colaborar (Amici, 2015), compartir recursos (Jensen, Vaish, \& Schmidt, 2014), asistir 
físicamente a otros y consolar (Warneken \& Tomasello, 2009) son manifestaciones prosociales que podrían ser comunes en los humanos.

Desde la aproximación cognitivo-afectiva, la empatía es un aspecto central que motiva la acción prosocial (Batson, 2011; Wang, Wang, Deng, \& Chen, 2019). Las personas ayudan por una autentica preocupación por el bienestar de otro compasión empática- (Batson, 2011; Kamas \& Preston, 2021; Padilla-Walker, Coyne, Collier, \& Nielson, 2015) o para aliviar los sentimientos de ansiedad, tensión o desagrado que pudieran provocar ver el sufrimiento de otros -perturbación propia- (Batson, 2011; Bierhoff \& Rohmann, 2004; Eisenberg, Fabes, \& Spinrad, 2006). Así mismo, el ser capaz de percibir los estados anímicos y emociones de otros -empatía cognoscitiva- (Carlo, McGinley, Hayes, Batenhorst, \& Wilkinson, 2007; Guo, Sun, Cai, Zhang, \& Song, 2019), el mantenerse tranquilo en situaciones emotivas -indiferencia- (Díaz-Loving, Andrade, \& Nadelsticher, 1986), así como tener la capacidad de regular las emociones desagradables que provoca la empatía (Carlo, Crockett, Wolff, \& Beal, 2012; Eisenberg et al. , 2006; Haroz, Murray, Bolton, Betancourt, \& Bass, 2013), propician el actuar prosocial. Desde esta perspectiva, la empatía es un rasgo de personalidad de la persona que promueve la conducta prosocial (Caprara, Alessandri, \& Eisenberg, 2012; Nook, Ong, Morelli, Mitchell, \& Zaki, 2016; Richaud \& Mesurado, 2016).

En relación con la aproximación psicosocial, desde la teoría del aprendizaje social el actuar prosocial se da por la interacción (Batson \& Powell, 2003; De La Cruz \& Rivera-Aragón, en prensa) e influencia (Calderón-Tena, Knight, \& Carlo, 2011; Penner et al., 2005) de aspectos individuales y contextuales. Las personas al interactuar con el medio aprenden (por medio del reforzamiento, castigo y modelamiento) cuáles son los estándares del comportamiento (Bandura, 1999). En el aspecto individual, el altruismo (Howard \& Piliavin, 2000), el bienestar personal (Sharma \& Tomer, 2018), las emociones positivas (Richaud \& Mesurado, 2016), el desarrollo personal (Clary et al., 1998) y las compensaciones materiales y monetarias (Dovidio et al., 2006) influyen para que una persona actúe prosocialmente. De igual forma, la edad condiciona la concepción y valoración de las acciones prosociales (Eisenberg et al., 2006). Con relación al contexto, observar que otros actúan prosocialmente (Nook et al., 2016), presenciar injusticias hacia otros (Van de Vyver \& Abrams, 2015), las experiencias de socialización (Garaigordobil, 2014) y valores culturales de colectivismo e individualismo (Giner-Torréns \& Kärtner, 2017; Mullen \& Skitka, 2009) influyen en la frecuencia y valoración del actuar prosocial.

Respecto a la evaluación de la conducta prosocial a través de escalas de autoinforme, Ladd y Profilet (1996) desarrollaron la Child Behavior Scale que evalúa conductas prosociales en el contexto escolar, y refieren a si un niño es amable, escucha, coopera, ayuda, consuela y se preocupa por sus compañeros de clase. Esta escala no especifica acciones concretas y considera situaciones conflictivas.

Méndez, Mendoza, Rodríguez y García (2015) validaron el inventario de Roche sobre Comportamientos Prosociales en el Contexto Escolar (1998, en Méndez et al., 2015) que evalúa conductas prosociales de empatía y consuelo verbal, escucha profunda, servicio físico y verbal, ayuda física y solidaridad en adolescentes. El énfasis de los reactivos recae en conductas propias del contexto escolar y deja de lado la situación y la motivación.

Balabanian y Lemos (2018) construyeron una escala en estudiantes adolescentes. El instrumento evalúa siete tipos de acciones prosociales: ayuda física, ayuda verbal, consuelo verbal, confirmación y valorización positiva del otro, solidaridad y presencia positiva y unidad. El contenido de las afirmaciones contempla conductas prosociales específicas y enfatiza algunas situaciones donde acontecen.

En adultos, Auné, Abal y Attoresi (2016) construyeron una escala para adultos que incluye comportamientos empáticos, altruismo, ayuda, compartir y donar. Las afirmaciones incluidas contemplan combinaciones entre conducta y motivación o situación y aunque está dirigida hacia adultos, gran parte de las acciones están enfocadas al contexto escolar universitario. Posteriormente, en una revisión de la escala, solo conservaron reactivos referentes a conductas (Auné et al., 2019). Es importante mencionar que, aunque los autores consideran la empatía como un 
comportamiento, Batson (2011) menciona que la empatía es más bien una motivación que promueve la respuesta prosocial.

Carlo y Randall (2002) desarrollaron la Prosocial Tendencies Measure en adultos. Esta escala quedó conformada por seis dimensiones: pública, anónima, emergencia, emocional, complaciente y altruista. La escala enfatiza las situaciones y motivaciones del actuar prosocial, más que acciones concretas.

Podemos observar que la ayuda instrumental (asistencia física) y emocional (consolar, escuchar) son comunes en la evaluación de niños, adolescentes y adultos (e. g. Auné et al., 2019; Ladd \& Profilet, 1996; Méndez et al., 2015). Sin embargo, a diferencia de niños y adolescentes, la evaluación en adultos considera conductas como donar y ser voluntario en organizaciones benéficas (e. g. Auné et al., 2019; Carlo \& Randall, 2002). Con respecto a estas diferencias, los adultos, al poseer más recursos, tienen mayor posibilidad de ayudar a otros (Penner, 2002) y son más propensos para comprometerse y dedicar esfuerzos para ser voluntarios (Wilson, 2000).

Respecto a la evaluación de la conducta prosocial, algunas escalas consideran únicamente conductas (Méndez et al., 2015), otras incluyen motivaciones y situaciones (Carlo \& Randall, 2002; Auné et al., 2016). Si contemplamos que una misma acción puede tener diferentes motivaciones y realizarse en distintas situaciones (Auné et al., 2016; De La Cruz \& Rivera-Aragón, en prensa; McGuire, 1994) las posibles combinaciones son muy numerosas. Ante esto, la evaluación independiente de las acciones prosociales resulta pertinente. Por lo que el propósito de este estudio fue desarrollar una escala de autoinforme para evaluar la conducta prosocial en adultos y obtener sus propiedades psicométricas (dimensiones que la componen, validez predictiva y confiabilidad).

\section{Método}

\section{Participantes}

Participaron 761 adultos de 18 a 75 años $(M=29.22, D E=12.21), 338$ hombres $(44.4 \%), 409$ mujeres $(53.7 \%)$ y $12(1.6 \%)$ no especificaron (ver Tabla 1). El criterio de inclusión fue habitar en la ciudad de México y área conurbada (al menos un año). El criterio de eliminación fue responder menos del $80 \%$ del instrumento. En todo momento se respetaron los aspectos del código ético del psicólogo para resguardar la dignidad de los participantes, confidencialidad de la información, consentimiento informado, no ofrecer incentivos inapropiados, informar posibles riesgos de la investigación y solicitar solo información básica pertinente para el estudio (Sociedad Mexicana de Psicología, 2009).

\section{Instrumentos}

Conducta prosocial: Con base en los resultados de un estudio exploratorio (De La Cruz \& Rivera-Aragón, en prensa) se redactaron 73 reactivos. En su redacción se empleó un lenguaje claro y simple. Además, se incluyeron reactivos redactados en dirección negativa para prevenir la tendencia de elegir solo opciones favorables. Los reactivos fueron sometidos a revisión por cinco jueces expertos en psicología social con amplia experiencia en la construcción de escalas psicológicas. Con el fin de procurar la validez de contenido y pertinencia teórica, los jueces evaluaron que en los reactivos fuera evidente que las acciones son para el beneficio de otro y abarcan variedad de manifestaciones prosociales, lográndose un $80 \%$ de acuerdo. La modalidad de respuesta fue a través de una escala tipo Likert con 5 opciones de respuesta (1=Nunca, 2=Algunas veces, 3=Regularmente, $\quad 4=$ Casi siempre, $5=$ Siempre).

Empatía: Se utilizó la Escala de Apreciación y Sensibilización Emocional (Díaz-Loving et al., 1986) desarrollada en México, la cual evalúa la empatía como rasgo de personalidad a través de 49 reactivos en escala con respuesta tipo Likert ( $1=$ Nunca a $5=$ Siempre) distribuidos en cuatro dimensiones: compasión empática $(\alpha=.83)$, perturbación propia $(\alpha=.89)$, empatía cognoscitiva $(\alpha=.79)$ e indiferencia $(\alpha=.77)$, los cuales explicaron el $41.3 \%$ de la varianza.

\section{Procedimiento}

Los instrumentos se aplicaron de forma individual o grupal en universidades y sitios de esparcimiento público en distintos sitios de la Ciudad de México de septiembre de 2019 a febrero 
Tabla 1. Datos sociodemográficos de la muestra

\begin{tabular}{|c|c|c|c|}
\hline \multicolumn{2}{|c|}{ Variable } & \multirow{2}{*}{$\begin{array}{c}\text { Frecuencia } \\
15\end{array}$} & \multirow{2}{*}{$\begin{array}{c}\text { Porcentaje } \\
1.97 \%\end{array}$} \\
\hline Escolaridad & Primaria & & \\
\hline & Secundaria & 50 & $6.57 \%$ \\
\hline & Bachillerato & 251 & $32.99 \%$ \\
\hline & Profesional & 408 & $53.62 \%$ \\
\hline & Posgrado & 28 & $3.67 \%$ \\
\hline & Sin estudios & 1 & $0.13 \%$ \\
\hline & No especificó & 8 & $1.05 \%$ \\
\hline \multirow[t]{3}{*}{ Estado civil } & Soltero & 575 & $75.69 \%$ \\
\hline & Casado & 134 & $17.6 \%$ \\
\hline & Unión Libre & 51 & $6.71 \%$ \\
\hline \multirow[t]{2}{*}{$\begin{array}{l}\text { Lugar de } \\
\text { residencia }\end{array}$} & $\begin{array}{l}\text { Estado de } \\
\text { México }\end{array}$ & 93 & $12.22 \%$ \\
\hline & $\begin{array}{l}\text { Ciudad de } \\
\text { México }\end{array}$ & 668 & $87.78 \%$ \\
\hline \multirow{5}{*}{$\begin{array}{l}\text { Situación } \\
\text { laboral }\end{array}$} & & & \\
\hline & Estudia & 356 & $46.79 \%$ \\
\hline & Trabaja & 397 & $52.17 \%$ \\
\hline & $\begin{array}{l}\text { Estudia y } \\
\text { trabaja }\end{array}$ & 4 & $0.52 \%$ \\
\hline & $\begin{array}{l}\text { No estudia ni } \\
\text { trabaja }\end{array}$ & 4 & $0.52 \%$ \\
\hline
\end{tabular}

de 2020. Se abordó a las personas de la siguiente forma:

Hola buenos días/buenas tardes, estoy realizando una investigación por parte de la facultad de psicología de la UNAM con relación a conductas realizadas en algún momento en la vida de las personas. Es un cuestionario y el tiempo para responderlo es de aproximadamente 20 minutos $i$ Tiene tiempo para apoyarme?

Si la persona aceptaba participar, el aplicador procedía a explicar las instrucciones:

La participación es anónima y confidencial, la información sólo será usada con fines estadísticos y científicos. Primero se encuentran una serie de preguntas sociodemográficas. A continuación, se presentan una serie de afirmaciones sobre distintas actividades que benefician a otros, por favor señale con una $X$ en qué medida las realiza. No existen respuestas correctas o incorrectas y es muy importante que responda todas las preguntas.

El aplicador permaneció alejado (al menos 3 metros) pero atento para resolver cualquier duda del participante.

\section{Resultados}

El análisis estadístico de la información se realizó con el software IBM SPSS, v. 24. Para el tratamiento de los valores perdidos, se empleó la opción excluir casos según lista, con el fin de tener el mismo número de casos validos en todos los resultados (George \& Mallery, 2001). Para el análisis psicométrico se siguieron los pasos propuestos por Nunnally y Bernstein (1995) y Reyes-Lagunes y García-y-Barragán (2008).

En primer lugar, se realizaron cinco análisis para evaluar el funcionamiento de los ítems en cuanto a su discriminación y aporte a la consistencia interna del instrumento para determinar qué reactivos serían sometidos al análisis factorial exploratorio: Primero se llevó a cabo un análisis de frecuencias para cada reactivo con el fin de verificar que todas las opciones de respuesta hayan sido elegidas por los participantes. Posteriormente, para comprobar el supuesto de normalidad de las variables, sé calculó el sesgo y curtosis de cada reactivo buscando valores entre \pm 1.5 (George \& Mallery, 2001). Después, para identificar si los reactivos distinguen entre niveles altos y bajos del rasgo latente, se empleó prueba $t$ de Student entre grupos extremos y la correlación entre el reactivo y el total de la prueba (índice de homogeneidad corregido) buscando valores superiores a .30. Por último, se obtuvo el alfa de Cronbach de la escala total y si al eliminar un reactivo éste aumentaba considerablemente el valor, la afirmación seria eliminada. Si un reactivo incumplía dos o más de los cinco criterios anteriores, seria descartado de los análisis posteriores. En este paso fueron eliminadas ocho afirmaciones (ver Tabla 2).

En segundo lugar, para obtener la validez de constructo de la escala, se empleó un análisis factorial exploratorio con factorización de ejes principales, considerando las recomendaciones y especificaciones de Osborne (2014). Las pruebas de Kaiser-Meyer-Olkin $(K M O=.91)$ y esfericidad de Bartlett $(p \leq .000)$, revelaron que la matriz era factorizable. Los reactivos que permanecieron en la escala presentaron comunalidades superiores a .3, de acuerdo con los criterios propuestos por Lloret, Ferreres, Hernández y Tomás (2014).

Para obtener la estructura factorial de la escala se eligió una rotación oblicua Promax con un valor Kappa $=4$ (Thompson, 2004) y debido a que las correlaciones entre factores mostraron valores superiores a .32, este método resultó adecuado (Ferrando \& Anguiano-Carrasco, 2010). Es importante mencionar que, al emplear una rotación 
Tabla 2. Análisis de discriminación y aporte a la consistencia interna de los reactivos de la escala de autoinforme de conducta prosocial para adultos

\begin{tabular}{|c|c|c|c|c|c|}
\hline Reactivo & Sesgo & Curtosis & $\begin{array}{l}\text { t de Student para } \\
\text { grupos extremos } \\
(p)\end{array}$ & $\begin{array}{c}\text { Correlación } \\
\text { con la escala } \\
\text { total }\end{array}$ & $\begin{array}{c}\alpha \text { si se } \\
\text { elimina el } \\
\text { elemento }\end{array}$ \\
\hline 1 Ayudo a otros (as) enseñando lo que sé & -.512 & -.640 & .000 & .347 & .881 \\
\hline 2 Aconsejo a otros (as) para apoyarlos con sus problemas & -.485 & -.610 & .000 & .388 & .881 \\
\hline 3 Ignoro a quienes necesitan que les ceda mi asiento & 1.324 & 1.308 & .654 & -.060 & .885 \\
\hline 4 Soy indiferente al compartir información sobre injusticia y desigualdad & 1.065 & .701 & .043 & .048 & .884 \\
\hline $\begin{array}{l}5 \text { Realizo servicio social de forma voluntaria para apoyar a mi } \\
\text { comunidad }\end{array}$ & 1.031 & .096 & .000 & .420 & .880 \\
\hline $\begin{array}{l}6 \text { Apoyo las marchas (paros) que luchan por una causa con la que estoy } \\
\text { de acuerdo }\end{array}$ & .559 & -.995 & .000 & .358 & .881 \\
\hline 7 Prefiero tirar cosas (ropa, cobijas, alimentos) a donarlas & 1.583 & 1.882 & .972 & -.018 & .885 \\
\hline 8 Escucho atentamente cuando una persona me cuenta sus problemas & -1.155 & 0.675 & .000 & .224 & .883 \\
\hline 9 Cedo el paso a las demás personas & -.728 & -.150 & .000 & .252 & .882 \\
\hline 10 Evito usar plásticos (bolsas, botellas) para cuidar el medio ambiente & .064 & -.833 & .000 & .342 & .881 \\
\hline 11 Compro en tiendas locales para apoyar su economía & -.180 & -.854 & .000 & .378 & .881 \\
\hline 12 Separo mi basura para cuidar el medio ambiente & -.459 & -1.049 & .000 & .240 & .883 \\
\hline 13 Desperdicio recursos (agua, luz), aunque afecte a otros & 1.524 & 2.371 & .089 & .060 & .884 \\
\hline $\begin{array}{l}14 \text { Evito usar desechables (platos, vasos, popotes) para cuidar el medio } \\
\text { ambiente }\end{array}$ & -.030 & -1.082 & .000 & .287 & .882 \\
\hline 15 Consuelo a otros (as) cuando lo necesitan & -.549 & -.615 & .000 & .404 & .881 \\
\hline 16 Dono ropa, cobijas o alimentos para los necesitados & .076 & -1.075 & .000 & .509 & .879 \\
\hline $\begin{array}{l}17 \text { Redondeo mis centavos al realizar compras, para ayudar a los } \\
\text { necesitados }\end{array}$ & .909 & -.301 & .000 & .309 & .882 \\
\hline 18 Prefiero quedarme con mis cosas (ropa, cobijas, alimentos) a donarlas & 1.313 & 1.144 & .960 & -.033 & .885 \\
\hline $\begin{array}{l}19 \text { Cuando otros (as) tienen problemas comparto mis experiencias para } \\
\text { consolarlos }\end{array}$ & -.298 & -.757 & .000 & .341 & .881 \\
\hline 20 Si alguien está pasando un mal rato, lo abrazo para confortarlo & -.213 & -1.173 & .000 & .451 & .880 \\
\hline 21 Participo por obligación (trabajo, escuela) en centros comunitarios & 1.161 & .475 & .000 & .214 & .883 \\
\hline 22 Infrinjo las normas de tránsito, aunque afecte a otros & 2.021 & 3.772 & .019 & .119 & .884 \\
\hline 23 Ignoro a las personas necesitadas que piden dinero & .784 & .250 & .515 & -.031 & .885 \\
\hline 24 Uso bicicleta o transporte público para cuidar el medio ambiente & -.081 & -1.342 & .000 & .171 & .884 \\
\hline 25 Ayudo a quienes me cuentan sus problemas & -.456 & -.748 & .000 & .437 & .880 \\
\hline $26 \mathrm{Si}$ a una persona se le cae dinero o algún objeto de valor, se lo entrego & -1.778 & 2.111 & .000 & .155 & .883 \\
\hline 27 Participo en colectas para apoyar buenas causas & .428 & -.845 & .000 & .480 & .880 \\
\hline 28 Cuido el medio ambiente participando en la limpieza de zonas verdes & .615 & -.697 & .000 & .473 & .880 \\
\hline 29 Dejo que cada quien realice su trabajo, aunque necesite ayuda & .625 & -.056 & .001 & .057 & .884 \\
\hline 30 Doy dinero a las personas que lo necesitan & .654 & -.194 & .000 & .418 & .881 \\
\hline 31 Ignoro a quienes me cuentan sus problemas & 1.888 & 2.840 & .863 & .017 & .885 \\
\hline 32 Soy voluntario (a) en labores de rescate. & 1.239 & .633 & .000 & .514 & .879 \\
\hline $\begin{array}{l}33 \text { Evito apoyar a otros (as) con sus labores, aunque tengan dificultades } \\
\text { para hacerlas }\end{array}$ & 1.341 & 1.778 & .688 & -.006 & .885 \\
\hline $34 \mathrm{Me}$ niego a donar sangre cuando alguien lo necesita & 1.488 & 1.432 & .396 & .021 & .885 \\
\hline 35 Evito prestar dinero a la gente, aunque lo necesite. & 1.009 & .473 & .354 & -.049 & .885 \\
\hline 36 Dono dinero para apoyar a personas que lo necesitan & .771 & -.096 & .000 & .461 & .880 \\
\hline 37 Evito desperdiciar agua por el bien común & -.860 & -.432 & .000 & .214 & .883 \\
\hline 38 Doy dinero a organizaciones que ayudan a animales de la calle & .735 & -.538 & .000 & .380 & .881 \\
\hline 39 Le aviso a una persona cuando se le cae dinero o algún objeto de valor & -1.676 & 1.797 & .000 & .163 & .883 \\
\hline 40 Evito que alguien se quede en mi casa, aunque lo necesite. & 1.257 & .648 & .558 & -.019 & .885 \\
\hline 41 Doy regalos a otros (as), aunque no sea una fecha especial & .372 & -.947 & .000 & .468 & .880 \\
\hline 42 Ignoro las colectas que apoyan buenas causas. & .982 & .501 & .086 & .002 & .885 \\
\hline 43 Dono juguetes a niños (as) necesitados. & .474 & -.923 & .000 & .548 & .879 \\
\hline $\begin{array}{l}44 \text { Apoyo a los trabajadores dando propina cuando pago por algún } \\
\text { servicio }\end{array}$ & -.530 & -.804 & .000 & .274 & .882 \\
\hline $\begin{array}{l}45 \text { Apoyo a otros (as) con sus labores cuando tienen dificultades para } \\
\text { hacerlas. }\end{array}$ & -.254 & -.811 & .000 & .470 & .880 \\
\hline 46 Ayudo a las personas dándoles trabajo & .716 & -.573 & .000 & .439 & .880 \\
\hline $\begin{array}{l}47 \text { Participo voluntariamente en campañas informativas de salud en } \\
\text { comunidades vulnerables. }\end{array}$ & 1.264 & .659 & .000 & .480 & .880 \\
\hline 48 Cedo mi asiento a personas que lo necesitan. & -.607 & -.817 & .000 & .297 & .882 \\
\hline 49 Ignoro las campañas de donación para apoyar a los necesitados. & .967 & .252 & .839 & -.012 & .885 \\
\hline 50 Ayudo a otros (as) a cargar cosas pesadas cuando lo necesitan. & -.207 & -1.091 & .000 & .396 & .881 \\
\hline 51 Dono sangre cuando puedo, para ayudar a otros (as). & .307 & -1.272 & .000 & .356 & .881 \\
\hline 52 Comparto alimentos (bebidas) con quien lo necesita. & -.196 & -1.082 & .000 & .510 & .879 \\
\hline 53 Ayudo a otros (as) a cruzar la calle. & .035 & -1.140 & .000 & .471 & .880 \\
\hline 54 Ignoro a las personas que necesitan ayuda para cruzar la calle. & 1.378 & 1.068 & .026 & .082 & .884 \\
\hline 55 Soy voluntario (a) en alguna asociación que busca ayudar a otros. & 1.061 & .004 & .000 & .460 & .880 \\
\hline 56 Presto dinero para ayudar a otros. & .550 & -.579 & .000 & .450 & .880 \\
\hline 57 Me rehúso a ser voluntario (a) en asociaciones que dan ayuda a otros. & 1.072 & .077 & .309 & -.040 & .886 \\
\hline 58 Le compro cosas a las personas para apoyarlas. & .303 & -.826 & .000 & .429 & .880 \\
\hline
\end{tabular}


Tabla 2. Análisis de discriminación y aporte a la consistencia interna de los reactivos de la escala de autoinforme de conducta prosocial para adultos (Cont.)

\begin{tabular}{|c|c|c|c|c|c|}
\hline Reactivo & Sesgo & Curtosis & $\begin{array}{l}\text { t de Student para } \\
\text { grupos extremos } \\
(p)\end{array}$ & $\begin{array}{l}\text { Correlación con } \\
\text { la escala total }\end{array}$ & $\begin{array}{c}\alpha \text { si se } \\
\text { elimina el } \\
\text { elemento }\end{array}$ \\
\hline $\begin{array}{l}59 \text { Respeto las normas de tránsito pensando en los } \\
\text { demás. }\end{array}$ & -.849 & -.283 & .000 & .223 & .883 \\
\hline 60 Si veo basura tirada la levanto, aunque no sea mía. & .100 & -1.135 & .000 & .455 & .880 \\
\hline 61 Sonrío a los demás para hacerlos sentir bien. & -.285 & -1.012 & .000 & .414 & .880 \\
\hline $\begin{array}{l}\text { 62Realizo actividades de voluntariado para apoyar a } \\
\text { quienes lo necesitan. }\end{array}$ & .777 & -.445 & .000 & .563 & .879 \\
\hline $\begin{array}{l}63 \text { Permito que alguien se quede en mi casa cuando lo } \\
\text { necesita. }\end{array}$ & .006 & -1.281 & .000 & .394 & .881 \\
\hline $\begin{array}{l}64 \text { Sin importarme la economía del vendedor, le } \\
\text { regateo. }\end{array}$ & 1.432 & 1.264 & .000 & .148 & .883 \\
\hline $\begin{array}{l}65 \text { Apoyo a alguien cuando tiene dificultades para } \\
\text { encontrar trabajo. }\end{array}$ & .265 & -.887 & .000 & .477 & .880 \\
\hline 66 Soy egoísta para compartir con otros lo que sé. & 1.783 & 2.875 & .030 & .089 & .884 \\
\hline $\begin{array}{l}67 \text { Comparto información para concientizar sobre la } \\
\text { injusticia y la desigualdad. }\end{array}$ & -.034 & -1.108 & .000 & .414 & .880 \\
\hline 68 Presto mis cosas cuando alguien las necesita. & -.222 & -.931 & .000 & .417 & .881 \\
\hline $\begin{array}{l}69 \text { Apoyo a otros (as) con su trabajo, aunque NO sea } \\
\text { mi responsabilidad. }\end{array}$ & .026 & -.966 & .000 & .492 & .880 \\
\hline 70 Participo voluntariamente en centros comunitarios. & 1.146 & .489 & .000 & .541 & .879 \\
\hline $\begin{array}{l}71 \text { Evito prestar mis cosas a otros, aunque las } \\
\text { necesiten. }\end{array}$ & 1.260 & 1.171 & .474 & -.006 & .885 \\
\hline $\begin{array}{l}72 \text { Evito ayudar a otros (as) cuando cargan cosas } \\
\text { pesadas. }\end{array}$ & 1.259 & .831 & .445 & -.003 & .885 \\
\hline 73 Indico a las personas como llegar a su destino & -.556 & -.703 & .000 & .337 & .881 \\
\hline
\end{tabular}

Nota. Los resultados en negritas no cumplen el criterio correspondiente

Para la conducta prosocial total el primer paso

oblicua, solo es interpretable el porcentaje total de la escala $(45.8 \%)$, esto porque los factores están correlacionados (Martínez, Hernández, \& Hernández, 2014). Por esta razón no se reportan porcentajes de varianza explicada por factor. La versión final de la escala quedó compuesta por 22 reactivos con una carga factorial mayor o igual 4. La consistencia interna de la escala se obtuvo con la prueba alfa de Cronbach (ver Tabla 3).

A continuación, se determinó el nombre para cada factor y su definición de acuerdo con la teoría (ver Tabla 4).

Con relación al análisis de validez predictiva, se empleó la Escala de Apreciación y Sensibilización Emocional (Díaz-Loving et al., 1986). Adicionalmente se calculó una medida global de conducta prosocial mediante el promedio de las puntuaciones de los 22 reactivos de la escala.

En primer lugar, se exploró la correlación Pearson entre empatía y conducta prosocial para detectar si existe multicolinealidad entre las variables. Puesto que las correlaciones fueron menores que .85 (Roth, 2012), se prosiguió con los análisis de regresión (ver Tabla 5).

A continuación, se realizaron análisis de regresión múltiple con el método pasos sucesivos considerando las correlaciones estadísticamente significativas (ver Tabla 6). de la regresión fue significativo ( $F$ $[1,757]=245.701, p<.001)$ e incluyó la compasión empática que explicó el $24.4 \%$ de la varianza $\left(R^{2}=.245, R^{2}\right.$ ajustada=.244). El segundo paso de la regresión fue significativo $(F[2,756]=143.931$, $p<.001)$ y se incorporó la empatía cognoscitiva $\left(R^{2}=.276, \quad R^{2} \quad\right.$ ajustada=.274, $\left.\Delta R^{2}=.031\right) . \quad$ En conjunto, la compasión empática y la empatía cognoscitiva explicaron el $27.4 \%$ de la varianza.

Con respecto al voluntariado, el primer paso de la regresión fue significativo ( $F$ $[1,757]=38.972, p<.001)$ e incluyó la compasión empática que explicó el $4.8 \%$ de la varianza $\left(R^{2}\right.$ $=.049, R^{2}$ ajustada=.048). El segundo paso de la regresión fue significativo $(F[2,756]=40.670$, $p<.001)$ y se incorporó la indiferencia $\left(R^{2}=.097\right.$, $R^{2}$ ajustada $\left.=.095, \Delta R^{2}=.048\right)$. En el tercer paso, la regresión fue significativa ( $F[3,755]=31.223$, $p<.001)$ y se incorporó la perturbación propia $\left(R^{2}=.110, \quad R^{2} \quad\right.$ ajustada $\left.=.107, \quad \Delta R^{2}=.013\right) . \quad$ En conjunto, la compasión empática, la indiferencia y la perturbación propia explicaron el $10.7 \%$ de la varianza.

En relación con la ayuda emocional, el primer paso de la regresión fue significativo ( $F$ $[1,757]=220.213, p<.001)$ e incluyó la compasión empática que explicó el $22.4 \%$ de la varianza $\left(R^{2}=.225, R^{2}\right.$ ajustada=.224). El segundo paso de la regresión fue significativo $(F[2,756]=147.162$, 
Tabla 3. Coeficientes de patrón de la escala de autoinforme de conducta prosocial para adultos

\begin{tabular}{|c|c|c|c|c|c|}
\hline \multirow{2}{*}{ Reactivo } & \multicolumn{4}{|c|}{ Factor } & \multirow{2}{*}{ Total } \\
\hline & 1 & 2 & 3 & 4 & \\
\hline Soy voluntario (a) en alguna asociación que busca ayudar a otros. & .881 & .023 & -.042 & -.109 & \\
\hline Participo voluntariamente en centros comunitarios. & .794 & .008 & .099 & -.055 & \\
\hline Realizo servicio social de forma voluntaria para apoyar a mi comunidad & .677 & .157 & -.133 & .018 & \\
\hline Realizo actividades de voluntariado para apoyar a quienes lo necesitan. & .609 & -.020 & .095 & .181 & \\
\hline $\begin{array}{l}\text { Participo voluntariamente en campañas informativas de salud en } \\
\text { comunidades vulnerables. }\end{array}$ & .564 & -.126 & .045 & .163 & \\
\hline Consuelo a otros (as) cuando lo necesitan & -.050 & .756 & .029 & .051 & \\
\hline Ayudo a quienes me cuentan sus problemas & -.101 & .696 & .081 & .070 & \\
\hline Si alguien está pasando un mal rato, lo abrazo para confortarlo & .099 & .624 & -.156 & .211 & \\
\hline Aconsejo a otros (as) para apoyarlos con sus problemas & .103 & .613 & .017 & -.040 & \\
\hline Escucho atentamente cuando una persona me cuenta sus problemas & .033 & .592 & .080 & -.214 & \\
\hline $\begin{array}{l}\text { Cuando otros (as) tienen problemas comparto mis experiencias para } \\
\text { consolarlos }\end{array}$ & -.008 & .551 & .023 & .008 & \\
\hline Apoyo a otros (as) con su trabajo, aunque NO sea mi responsabilidad. & .040 & .016 & 687 & .023 & \\
\hline Ayudo a otros (as) a cargar cosas pesadas cuando lo necesitan. & -.015 & -.026 & .639 & .114 & \\
\hline Comparto alimentos (bebidas) con quien lo necesita. & .004 & -.008 & .635 & -.134 & \\
\hline Presto mis cosas cuando alguien las necesita. & -.008 & .231 & .578 & -.101 & \\
\hline Apoyo a otros (as) con sus labores cuando tienen dificultades para hacerlas. & -.114 & .104 & .511 & .162 & \\
\hline Presto dinero para ayudar a otros. & .114 & .010 & .460 & .067 & \\
\hline Dono ropa, cobijas o alimentos para los necesitados & -.025 & .149 & -.040 & .610 & \\
\hline Cuido el medio ambiente participando en la limpieza de zonas verdes & .194 & -.102 & -.104 & .592 & \\
\hline Participo en colectas para apoyar buenas causas & .125 & .025 & -.016 & .571 & \\
\hline Dono dinero para apoyar a personas que lo necesitan & .049 & -.062 & .149 & .482 & \\
\hline Dono juguetes a niños (as) necesitados. & .118 & -.051 & .197 & .447 & \\
\hline \multicolumn{6}{|l|}{ Correlación entre factores } \\
\hline 1. Voluntariado & - & .127 & .334 & .638 & \\
\hline 2. Ayuda emocional & - & - & .614 & .413 & \\
\hline 3. Ayuda instrumental & - & - & - & .606 & \\
\hline 4. Donación & - & - & - & - & \\
\hline Número de reactivos & 5 & 6 & 6 & 5 & 22 \\
\hline Varianza explicada (\%) & & & & & 45.871 \\
\hline Media & 2.037 & 3.666 & 3.205 & 2.614 & 2.931 \\
\hline Desviación estándar & .944 & .818 & .838 & .878 & .651 \\
\hline Alfa de Cronbach & .846 & .813 & .798 & .749 & .890 \\
\hline
\end{tabular}

Nota. Los pesos factoriales por afirmación están resaltados en negritas de acuerdo con el factor al que pertenecen. 1=Voluntariado, 2=Ayuda emocional, 3=Ayuda instrumental, 4=Donación.

Tabla 4. Nombre y definición de los factores

\begin{tabular}{ll}
\hline \multicolumn{1}{c}{ Factor } & \multicolumn{1}{c}{ Definición } \\
\hline Voluntariado & $\begin{array}{l}\text { Participación voluntaria y planificada en organizaciones o grupos que buscan beneficiar a los } \\
\text { necesitados. }\end{array}$ \\
Ayuda emocional & $\begin{array}{l}\text { Acciones que brindan apoyo verbal, atención y compañía hacia quienes tienen un problema. } \\
\text { Cyuda instrumental } \\
\text { Conductas que proporcionan asistencia física o material a quienes lo necesitan. } \\
\text { Entrega voluntaria de recursos materiales o humanos para el beneficio de los demás. }\end{array}$ \\
\hline
\end{tabular}

Tabla 5. Correlaciones entre la escala de autoinforme de conducta prosocial para adultos y la EASE

\begin{tabular}{|c|c|c|c|c|}
\hline Variable & Compasión Empática & Empatía Cognoscitiva & Perturbación Propia & indiferencia \\
\hline Voluntariado & $.221 * *$ & $.113^{* *}$ & $.158 * *$ & $.165 * *$ \\
\hline Ayuda Emocional & $.475 * *$ & $.381 * *$ & $.154 * *$ & $-.093 *$ \\
\hline Ayuda Instrumental & $.445 * *$ & $.289 * *$ & $.101 * *$ & -.053 \\
\hline Donación & $.336 * *$ & $.212 * *$ & $.147 * *$ & .022 \\
\hline $\begin{array}{l}\text { Conducta Prosocial } \\
\text { Total }\end{array}$ & $.495 * *$ & $.334 * *$ & $.185 * *$ & .11 \\
\hline
\end{tabular}

Nota. Las correlaciones resaltadas en negritas son estadísticamente significativas. $* * p<.01 ; * p<.05$.

$p<.001)$ y se incorporó la empatía cognoscitiva $\left(R^{2}=.280, \quad R^{2}\right.$ ajustada $\left.=0.278, \Delta R^{2}=.055\right) . \quad$ En conjunto, la compasión empática y la empatía cognoscitiva explicaron el $27.8 \%$ de la varianza.

Para la ayuda instrumental, en el primer paso la regresión fue significativo $(F[1,757]=187.254$, $p<.001)$ e incluyó la compasión empática que explicó el $19.7 \%$ de la varianza $\left(R^{2}=.198, R^{2}\right.$ ajustada=.197). El segundo paso de la regresión fue significativo $(F[2,756]=106.439, p<.001) \mathrm{y}$ se incorporó la empatía cognoscitiva $\left(R^{2}=.220, R^{2}\right.$ ajustada=.218, $\left.\Delta R^{2}=.021\right)$. En el tercer paso, la 
Tabla 6. Análisis de regresión por pasos sucesivos para las dimensiones de empatía que predicen la conducta prosocial

\begin{tabular}{|c|c|c|c|c|c|}
\hline \multirow{2}{*}{ Dimensiones } & \multirow{2}{*}{$\mathrm{B}$} & \multirow{2}{*}{$\mathrm{EE}$} & \multirow{2}{*}{$\beta$} & \multicolumn{2}{|c|}{ Intervalo de confianza $95 \%$} \\
\hline & & & & Mínimo & Máximo \\
\hline \multicolumn{6}{|l|}{ Conducta prosocial total } \\
\hline Compasión empática & .495 & .037 & $.433 * * *$ & .421 & .568 \\
\hline Empatía cognoscitiva & .177 & .031 & $.186 * * *$ & .116 & .239 \\
\hline \multicolumn{6}{|l|}{ Voluntariado } \\
\hline Compasión empática & .372 & .063 & $.224 * * *$ & .247 & .496 \\
\hline Indiferencia & .313 & .045 & $.249 * * *$ & .225 & .402 \\
\hline Perturbación propia & .245 & .073 & $.130 * * *$ & .102 & .389 \\
\hline \multicolumn{6}{|l|}{ Ayuda emocional } \\
\hline Compasión empática & .563 & .047 & $.392 * * *$ & .471 & .655 \\
\hline Empatía cognoscitiva & .298 & .039 & $.248 * * *$ & .221 & .375 \\
\hline \multicolumn{6}{|l|}{ Ayuda instrumental } \\
\hline Compasión empática & .646 & .055 & $.439 * * *$ & .539 & .753 \\
\hline Empatía cognoscitiva & .190 & .042 & $.154 * * *$ & .108 & .271 \\
\hline Perturbación propia & -.179 & .059 & $-.107 * *$ & -.295 & -.063 \\
\hline \multicolumn{6}{|l|}{ Donación } \\
\hline Compasión empática & .462 & .056 & $.3 * * *$ & .352 & .571 \\
\hline Empatía cognoscitiva & .140 & .047 & $.108 * *$ & .048 & .231 \\
\hline
\end{tabular}

Nota. ${ }^{*} p \leq .05 ;{ }^{* *} p \leq .01 ;{ }^{* * *} p \leq .001$

regresión fue significativa $(F \quad[3,755]=74.759$, $p<.001)$ y se incorporó la perturbación propia $\left(R^{2}=.229, R^{2}\right.$ ajustada=.226, $\left.\Delta R^{2}=.009\right)$. En total, la compasión empática, la empatía cognoscitiva y la perturbación propia explicaron el $22.6 \%$ de la varianza.

Finalmente, para la donación el primer paso de la regresión fue significativo $(F$ $[1,757]=96.142, p<.001)$ e incluyó la compasión empática que explicó el $11.2 \%$ de la varianza $\left(R^{2}=.113, R^{2}\right.$ ajustada=.112). El segundo paso de la regresión fue significativo $(F[2,756]=53.084$, $p<.001)$ e incorporó la empatía cognoscitiva $\left(R^{2}=.123, \quad R^{2} \quad\right.$ ajustada $\left.=.121, \quad \Delta R^{2}=.01\right) . \quad$ En conjunto, la compasión empática y la empatía cognoscitiva explicaron el $12.1 \%$ de la varianza.

\section{Discusión}

El propósito de este estudio fue desarrollar una escala de autoinforme para evaluar la conducta prosocial en adultos de la ciudad de México y área conurbada y obtener sus propiedades psicométricas. La escala quedó conformada cuatro dimensiones: voluntariado, ayuda emocional, ayuda instrumental y donación.

Respecto a las propiedades psicométricas de la escala, esta presenta un nivel adecuado de validez (Martínez et al., 2014), además, su correspondencia con la Escala de Apreciación y
Sensibilización Emocional es evidencia de validez predictiva, lo cual muestra que el instrumento es apropiado para evaluar las manifestaciones prosociales propuestas. Así mismo, los valores alfa de Cronbach son adecuados (Oviedo \& Campo, 2005) por lo que el instrumento es capaz de obtener mediciones consistentes.

Respecto a las dimensiones de la escala. El voluntariado es un comportamiento prosocial que requiere niveles altos de compromiso y esfuerzo (Wilson, 2000). La participación comunitaria para fomentar el bienestar social (De La Cruz \& Rivera-Aragón, en prensa) y el ser voluntario en organizaciones benéficas (Auné et al., 2019) son manifestaciones prosociales más propias de los adultos. Esto puede deberse a que, por un lado, la posesión de recursos (tiempo o dinero) facilitan su realización (Penner, 2000). Por otro lado, el voluntariado al poder ser una actividad institucionalizada existe la posibilidad de ser una labor remunerada (Wilson, 2000), es decir, puede ser la actividad laboral de la persona.

La segunda dimensión ayuda emocional son expresiones prosociales que parecen ser más comunes en culturas colectivistas, puesto que enfatizan la reciprocidad y el compromiso en las relaciones interpersonales (Mullen \& Skitka, 2009) y se manifiestan tanto en niños (Ladd \& 
Profilet, 1996), adolescentes (Mendez et al., 2015) y adultos (Auné et al., 2019; De La Cruz \& Rivera-Aragón, en prensa).

El factor de ayuda instrumental es una conducta prosocial común en todos los grupos etarios (Giner-Torréns \& Kärtner, 2017; Méndez et al., 2015; De La Cruz \& Rivera-Aragón, en prensa). Este tipo de acciones al requerir poco esfuerzo y bajo costo para realizarse (McGuire,1994) son manifestaciones prosociales susceptibles de presentarse de manera cotidiana (De La Cruz \& Rivera-Aragón, en prensa) y las personas de sociedades colectivistas las realizan con mayor frecuencia (Giner-Torréns \& Kärtner, 2017).

La dimensión de donación es considerada principalmente en adultos (Auné et al., 2019; Carlo \& Randall, 2002; De La Cruz \& RiveraAragón, en prensa) y podrían ser una manifestación más común en esta población, puesto que poseen los recursos financieros para hacerlo (Penner, 2002). No obstante, la donación parece estar influida en gran medida por aspectos contextuales, como el presenciar la generosidad de otras personas (Nook et al., 2016) o ser testigo de alguna injusticia hacia otros (Van de Vyver \& Abrams, 2015).

Estas cuatro dimensiones que componen la escala son consistentes con las propuestas biológicas y evolutivas sobre la predisposición genética (Fortuna \& Knafo, 2014) y tendencia innata a realizar conductas prosociales como la asistencia física, compartir recursos, consolar y colaborar (Amici, 2015; Jensen et al., 2014). Si bien las conductas prosociales que emergen en los primeros años de vida esencialmente coinciden con las acciones que conforman la presente escala, aspectos relacionados con el desarrollo biológico (Eisenberg et al., 2006), la cultura y la socialización (Warneken \& Tomasello, 2009) influyen en la forma de expresarlas. Es decir, mediante un proceso un aprendizaje social las personas desarrollan formas particulares de prosocialidad (Calderón-Tena et al., 2011). En este sentido, los resultados del presenten estudio muestran que para los adultos la expresión de la ayuda instrumental contempla acciones de asistencia física y material (e. g. cargar cosas pesadas, prestar objetos o dinero y apoyar a otros en sus labores), el compartir recursos se expresa mediante la entrega de bienes materiales para el beneficio de otros (e. g. donar objetos, alimentos o dinero), consolar se expresa por medio de apoyo verbal, atención y compañía (e. g. abrazar, aconsejar y escuchar los problemas de otros) y colaborar se manifiesta en la participación voluntaria en organizaciones benéficas.

Con relación a la validez predictiva se empleó una medida de empatía disposicional puesto que ha demostrado consistentemente ser predictor de la conducta prosocial (Caprara et al., 2012; Nook et al., 2016; Richaud \& Mesurado, 2016). Los resultados muestran que aquellas personas con una mayor capacidad de sentir empatía están más dispuestas para ayudar a otros, siendo la compasión empática el principal predictor. En este sentido la compasión y preocupación por el bienestar de otros son el aspecto con mayor influencia en la disposición para actuar prosocialmente (Batson, 2011; Kamas \& Preston, 2021; Padilla-Walker et al., 2015). El segundo elemento empático más consistente en la predicción de la conducta prosocial es la empatía cognoscitiva, lo cual muestra la importancia que tiene la capacidad de percibir y entender el estado anímico de los demás en las acciones prosociales de las personas (Carlo et al., 2007; Guo et al., 2019).

Otro aspecto afectivo empático presente es la perturbación propia, específicamente como predictor del voluntariado y la ayuda instrumental. Con respecto al voluntariado, sentir ansiedad, tensión o desagrado por la desgracia de otros favorece que las personas se involucren en organizaciones benéficas. En este aspecto, experimentar sensaciones desagradables ante el sufrimiento de otros influyen en la ayuda (Batson, 2011; Bierhoff \& Rohmann, 2004). En contraste, para la ayuda instrumental una menor presencia de perturbación propia favorece la procuración de asistencia física o material, es decir, las emociones negativas que provoca la empatía pueden disminuir la ayuda (Batson, 2011; Eisenberg et al., 2006).

Finalmente, la indiferencia, el sentir tranquilidad (o desinterés) ante los problemas de otros promueve el voluntariado. Este hallazgo puede parecer incongruente, sobre todo si consideramos que la compasión empática y la perturbación propia también son predictores del 
voluntariado. En este sentido, Díaz-Loving et al. (1986) mencionan que pueden existir situaciones en las que sea necesario permanecer indiferente y tranquilo para poder ayudar a otros. Si bien la preocupación empática es promotora de la ayuda (Batson, 2011; Kamas \& Preston, 2021; PadillaWalker et al., 2015), la perturbación propia si es muy elevada puede inhibir la respuesta prosocial (Batson, 2011; Eisenberg et al., 2006) por lo que ser capaz de regular emociones negativas es necesario para poder ayudar (Carlo et al., 2012; Eisenberg et al., 2006; Haroz et al., 2013).

En relación con las limitaciones del estudio, la presente investigación se enfocó en obtener una escala de autoinforme que evalúa únicamente conductas, sin embargo, la motivación y situación son elementos que forman parte del constructo (De La Cruz \& Rivera-Aragón, en prensa), por lo que el siguiente paso de la investigación es continuar con la construcción y análisis psicométricos de las escalas de motivaciones y situaciones prosociales. En futuras investigaciones es necesario realizar un análisis factorial confirmatorio para proporcionar mayor evidencia de la validez de constructo de la escala.

En conclusión, las dimensiones obtenidas son similares a las de otras escalas. Con aquellas dirigidas a niños y adolescentes coinciden la ayuda emocional (Balabanian \& Lemos, 2018) e instrumental (Méndez et al., 2015). En el caso de las escalas para adultos, que están dirigidas al contexto escolar (Auné et al., 2016) o están centradas en la motivación para realizar las conductas (Carlo \& Randall, 2002), coinciden la ayuda emocional, instrumental, voluntariado y donación (Auné et al., 2019; Carlo \& Randall, 2002). Si bien hay equivalencias, el aporte del presente estudio fue obtener un instrumento con sensibilidad cultural para evaluar la conducta prosocial en población abierta de la ciudad de México, es decir, es una escala que toma en cuenta la naturaleza social e idiosincrática de la conducta prosocial (House et al., 2020; Penner et al., 2005).

\section{Referencias}

Aknin, L. B., Whillans, A. V., Norton, M. I., \& Dunn, E. W. (2019). Happiness and Prosocial Behavior. En J. F. Helliwell, R. Layard \& J. D. Sachs (Eds.), World Happiness Report 2019 (pp. 67-86). New York, EUA: Sustainable Development Solutions Network.

Amici, F. (2015). The evolution and development of human cooperation. Interaction Studies, 16(3), 383-418.

https://doi.org/10.1075/is.16.3.03ami.

Auné, S. E., Abal, F. J., \& Attorresi, H. F. (2016). Diseño y construcción de una escala de conducta prosocial para adultos. Revista Iberoamericana de Diagnóstico y Evaluación - e Avaliação Psicológica, 42(2),15-25. https://doi.org/10.21865/RIDEP42_15.

Auné, S. E., Abal, F. J., \& Attorresi, H. F. (2019). $\mathrm{La}$ estructura de la conducta prosocial. Su aproximación mediante el modelo bifactorial de la Teoría de la Respuesta al Ítem Multidimensional. Liberabit, 25(1), 41-56. http://dx.doi.org/10.24265/liberabit.2019.v25n 1.04 .

Balabanian, C., \& Lemos, V. (2018). Desarrollo y estudio psicométrico de una Escala para Evaluar Conducta Prosocial en Adolescentes. Revista Iberoamericana de Diagnóstico y Evaluación - e Avaliação Psicológica, 3(43), 177-188. https://doi.org/10.21865/RIDEP48.3.15.

Bandura, A. (1999). Social cognitive theory: An agentic perspective. Asian Journal of Social Psychology, 2, 21-41.

https://doi.org/10.1111/1467-839X.00024.

Batson, C. D., \& Powell, A. A. (2003). Altruism and prosocial behavior. En I. B. Weiner, T. Millon \& M. J. Lerner (Eds.), Handbook of Psychology. Volume 5 Personality and social psychology (pp. 463-484). New Jersey, EUA: John Wiley \& Sons, Inc.

Batson, C. D. (2011). Altruism in humans. Oxford, UK: Oxford University Press.

Bierhoff, H.-W., \& Rohmann, E. (2004). Altruistic personality in the context of the empathy-altruism hypothesis. European Journal of Personality, 18(4), 351-365. https://doi.org/10.1002/per.523. 
Bravo, C., \& Reyes-Lagunes, I. (2009). Aproximaciones metodológicas en la medición de la conducta prosocial en niños de edad escolar. Revista Iberoamericana de Diagnóstico y Evaluación - e Avaliação Psicológica, 1(27), 29-44. Recuperado de: https://dialnet.unirioja.es/servlet/articulo?codi go $=4539877$.

Calderón-Tena, C. O., Knight, G. P. , \& Carlo, G. (2011). The socialization of prosocial behavioral tendencies among Mexican american adolescents: The role of familism values. Cultural Diversity and Ethnic Minority Psychology, 17(1), 98-106. https://doi.org/10.1037/a0021825.

Caprara, G. V., Alessandri, G., \& Eisenberg, N. (2012). Prosociality: The contribution of traits, values, and self-efficacy beliefs. Journal of Personality and Social Psychology, 102(6), 1289-1303. https://doi.org/10.1037/a0025626.

Carlo, G., \& Randall, B. A. (2002). The development of a measure of prosocial behaviors for late adolescents. Faculty Publications Department of Psychology, 70. https://doi.org/10.1023/A:1014033032440.

Carlo, G., Crokett, L. J., Wolff, J. M., \& Beal, S. J. (2012). The role of emotional reactivity, self-regulation, and puberty in adolescents' prosocial behaviors. Social Development, 21(4), 667-685.

https://doi.org/10.1111/j.14679507.2012.00660.x.

Carlo, G., McGinley, M., Hayes, R., Batenhorst, C., \& Wilkinson, J. (2007). Parenting styles or practices? Parenting, sympathy, and prosocial behaviors among adolescents. The Journal of Genetic Psychology, 168(2), 147-176. doi:10.3200/GNTP.168.2.147-176.

Clary, E. G., Snyder, M., Ridge, R. D., Copeland, J., Stukas, A. A., Haugen, J., \& Miene, P. (1998). Understanding and assessing the motivations of volunteers: A functional approach. Journal of Personality and Social Psychology, 74(6), 1516-1530. https://doi.org/10.1037/0022-3514.74.6.1516.

Collett, J. L., \& Morrissey, C. A. (2007). The social psychology of generosity: The state of current interdisciplinary research. Report for the John Templeton Foundation Generosity
Planning Project. Recuperado de https://www.researchgate.net/publication/242 082101_The_Social_Psychology_of_Generosi ty_The_State_of_Current_Interdisciplinary_R esearch.

De La Cruz, M. A., \& Rivera-Aragón, S. (en prensa). La conducta prosocial en adultos: Conceptuación y valoración. En C. ArmentaHurtarte (Ed.), La Psicología Social en México, Ciudad de México: Editorial de la Universidad Iberoamericana.

Díaz-Loving, R., Andrade, P., \& Nadelsticher, A. (1986). Desarrollo de la Escala Multidimensional de Empatía (EASE). Revista de Psicología Social y Personalidad, 2(1), 3-11.

Dovidio, J. F., Piliavin, J. A., Schroeder, D. A., \& Penner, L. A. (2006). The social psychology of prosocial behavior. New Jersey: Laurence Erlbaum Associates.

Eisenberg, N., Fabes, R. A., \& Spinrad, T. L. (2006). Prosocial development. En N. Eisenberg, W. Damon \& R. M. Lerner (Eds), Handbook of child psychology, (pp. 701-778). Nueva York: John Wiley \& Sons, Inc.

Eisenberg, N., \& Spinrad, T. (2014). Multidimensionality of prosocial behavior: Rethinking the conceptualization and development of prosocial behavior. En L. M. Walker \& G. Carlo (Eds.), Prosocial Development: A Multidimensional Approach (pp. 17-42). New York, EUA. Oxford University Press.

Eisenberg, N., Spinrad, T. L., \& Knafo, A. (2015). Prosocial development. En M. E. Lamb \& R. M. Lerner (Eds.), Handbook of child psychology and developmental science: Socioemotional processes (pp. 610-656). Hoboken, Nueva Jersey, Estados Unidos: John Wiley \& Sons Inc.

Ferrando, P. J., \& Anguiano-Carrasco, C. (2010). El análisis factorial como técnica de investigación en psicología. Papeles del Psicólogo, 31(1), 18-33. Recuperado de https://dialnet.unirioja.es/servlet/articulo?codi go $=3150810$.

Fortuna, K., \& Knafo, A. (2014). Parental and genetic contributions to prosocial behavior during childhood. En L. M. Padilla-Walker, G. Carlo (Eds.), Prosocial Development: A 
Multidimensional Approach (pp.70-89).

Nueva York, EUA: Oxford University Press.

Garaigordobil, M. (2014). Conducta prosocial: El papel de la cultura, la familia, la escuela y la personalidad. Revista Mexicana de Investigación en Psicología, 6(2), 146-157. Recuperado de https://www.medigraphic.com/cgi$\mathrm{bin} / \mathrm{new} /$ resumen.cgi?IDARTICULO $=70550$.

Gebauer, J. E., Riketta, M., Broemer, P., \& Maio, G. R. (2008). Pleasure and pressure based prosocial motivation: Divergent relations to subjective well-being. Journal of Research in Personality, 42, 399-420. https://doi.org/10.1016/j.jrp.2007.07.002.

George, D., \& Mallery, M. P. (2001). SPSS for Windows step by step: A simple guide and reference. Boston, MA: Allyn \& Bacon.

Giner-Torréns, M., \& Kärtner, J. (2017). The influence of socialization on early helping from a cross-cultural perspective. Journal of Cross-Cultural Psychology, 48(3), 353-368. https://doi.org/10.1177/0022022117690451.

Grant, A. \& Dutton, J. (2012). Beneficiary or benefactor: Are people more prosocial when they reflect on receiving or giving? Psychological Science, 23(9), 1033-1039. https://doi.org/10.1177/0956797612439424.

Grusec, J. E., Hastings, P., \& Almas, A. (2011). Prosocial Behavior. En P. K. Smith \& C. H. Hart (Eds.). The Wiley-Blackwell Handbook of Childhood Social Development (pp. 549566). Inglaterra, Reino Unido: John Wiley \& Sons Ltd.

Guo, Q., Sun, P., Cai, M., Zhang, X., \& Song, K. (2019). Why are smarter individuals more prosocial? A study on the mediating roles of empathy and moral identity. Intelligence, 75, 18. https://doi.org/10.1016/j.intell.2019.02.006.

Haroz, E. E., Murray, L. K., Bolton, P., Betancourt, T., \& Bass, J. K. (2013). Adolescent resilience in Northern Uganda: The role of social support and prosocial behavior in reducing mental health problems. Journal of Research on Adolescence, 23, 138148.

https://doi.org/10.1111/j.15327795.2012.00802.x.

House, B. R., Kanngiesser, P., Barrett, H. C. et al. (2020). Universal norm psychology leads to societal diversity in prosocial behaviour and development. Nature Human Behaviour 4, 3644.

https://doi.org/10.1038/s41562-019-0734-z.

Howard, J. A., \& Piliavin, J. A. (2000). Altruism. En E. F. Borgatta y R. J. V. Montgomery (Eds.), Encyclopedia of Sociology (pp, 114120). Nueva York: Macmillian.

Jensen, K., Vaish, A., \& Schmidt, M. (2014). The emergence of human prosociality: Aligning with others through feelings, concerns, and norms. Frontiers in Psychology, 5, 1-16. https://doi.org/10.3389/fpsyg.2014.00822.

Kamas, L., \& Preston, A. (2021). Empathy, gender and prosocial behavior. Journal of Behavioral and Experimental Economics, 92. https://doi.org/10.1016/j.socec.2020.101654.

Ladd, G. W., \& Profilet, S. M. (1996). The Child Behavior Scale: A teacher-report measure of young children's aggressive, withdrawn, and prosocial behaviors. Developmental Psychology, 32(6), 1008-1024.

https://doi.org/10.1037/0012-1649.32.6.1008.

Laninga-Wijnen, L., Harakeh, Z., Dijkstra, J. K., Veenstra, R., \& Vollebergh, W. (2018). Aggressive and prosocial peer norms: Change, stability and associations with adolescent aggressive and prosocial behavior development. Journal of Early Adolescence, 38(2), 178-203.

https://doi.org/10.1177/0272431616665211.

Lapo, M., \& Bustamante, M. (2018). Incidencia del clima organizacional y de las actitudes laborales en el comportamiento prosocial de los profesionales de la salud del Guayas Ecuador. Información Tecnológica, 29(5), 245-258. http://dx.doi.org/10.4067/S071807642018000500245.

Lloret, S., Ferreres, A., Hernández, A., \& Tomás, I. (2014). El análisis factorial exploratorio de los ítems: Una guía práctica, revisada y actualizada. Anales de Psicología, 30(3), 1151-1169. https://dx.doi.org/10.6018/analesps.30.3.199361

Martínez, M. R., Hernández, M. V., \& Hernández, M. J. (2014). Psicometría. España, Madrid: Alianza Editorial, S. A.

McGuire, A. (1994). Helping behaviors in the natural environment: Dimensions and correlates of helping. Personality and Social 
Psychology Bulletin, 20(1), 45-56. https://doi.org/10.1177/0146167294201004.

Méndez, F., Mendoza, C., Rodríguez, L., \& García, M. (2015). Conducta prosocial en alumnos de secundaria: Validación de una Escala Prosocial. VERTIENTES Revista Especializada en Ciencias de la Salud, 18(2), 9-16. Recuperado de:

http://www.revistas.unam.mx/index.php/vertie ntes/article/view/56488.

Moradi, S., Van Quaquebeke, N., \& Hunter, J. A. (2018). Flourishing and prosocial behaviors: A multilevel investigation of national corruption level as a moderator. PLOS ONE 13(7).

https://doi.org/10.1371/journal.pone.0200062.

Mujcic, R., \& Leibbrandt, A. (2017). Indirect reciprocity and prosocial behaviour: Evidence from a natural field experiment. The Economic Journal, 128, 1683-1699. https://doi.org/10.1111/ecoj.12474.

Mullen, E., \& Skitka, L. J. (2009). Comparing Americans' and Ukrainians' allocations of public assistance: The role of affective reactions in helping behavior. Journal of Cross-Cultural Psychology, 40, 301-318. https://doi.org/10.1177/0022022108328916.

Nook, E., Ong, D. C., Morrelli, S. A., Mitchel, J. P., \& Zaki, J. (2016). Prosocial conformity: Prosocial norms generalize across behavior and empathy. Personality and Social Psychology Bulletin, 1-18. https://doi.org/10.1177/0146167216649932.

Nunnally, J. C., \& Bernstein, I. J. (1995). Teoría psicométrica ( $3^{\mathrm{a}}$ ed). México, D.F.: Editorial McGrawHill Latinoamericana.

Osborne, J. W. (2014). Best Practices in exploratory factor analysis. California. USA: CreateSpace Independent Publishing Platform.

Oviedo, C., \& Campo, A. (2005). Aproximación al uso del coeficiente alfa de Cronbach. Revista Colombiana de Psiquiatría, 34(4), 572-580. Recuperado de: https://www.redalyc.org/pdf/806/80634409.pdf Padilla-Walker, L. M., Coyne, S. M., Collier, K. M., \& Nielson, M. G. (2015). Longitudinal relations between prosocial television content and adolescents' prosocial and aggressive behavior: The mediating role of empathic concern and self-regulation. Developmental Psychology, 51(9), 1317-1328. https://doi.org/10.1037/a0039488.

Palomar, J., \& Victorio, A. (2018). Predictores y correlatos del comportamiento prosocial de adolescentes mexicanos. Interdisciplinaria, 35(2), 495-509. Recuperado de: https://www.redalyc.org/journal/180/1805878 5015/html/.

Penner, L. A. (2002). Dispositional and organizational influences on sustained volunteerism: An interactionist perspective. Journal of Social Issues, 58(3), 447-467. https://doi.org/10.1111/1540-4560.00270.

Penner, L. A., Dovidio, J. F., Pilavin, J. A., \& Schroeder, D. A. (2005). Prosocial behavior: Multilevel perspectives. Annual Review of Psychology, 56, 365-392.

https://doi.org/10.1146/annurev.psych.56.091 103.070141.

Reyes Lagunes, I., \& García y Barragán, L. (2008). Hacia un procedimiento de validación psicométrica culturalmente relevante. $L a$ psicología social en México, 12, 625-630. Recuperado de:

https://www.researchgate.net/publication/259

191173_PROCEDIMIENTO_DE_VALIDAC ION_PSICOMETRICA_CULTURALMENT E_RELEVANTE_UN_EJEMPLO.

Richaud, M. C., \& Mesurado, B. (2016). Las emociones positivas y la empatía como promotores de las conductas prosociales e inhibidores de las conductas agresivas. Acción Psicológica, 13(2), 31-42.

https://dx.doi.org/10.5944/ap.13.2.17808.

Roth, E. (2012). Análisis multivariado en la investigación psicológica: Modelado Predictivo y Causal con SPSS y AMOS. La Paz. Bolivia: SOIPA Ltda.

Sharma, S., \& Tomer, S. (2018). Psychosocial antecedents of prosocial behavior and its relationship with subjective well-being in adolescents. Indian Journal of Positive Psychology, 9(1), 14-21. https://doi.org/10.15614/ijpp.v9i01.11736.

Sheldon, K. M., Wineland, A., Venhoeven, L., \& Osin, E. (2016). Understanding the motivation of environmental activists: A comparison of self-determination theory and functional 
motives theory. Ecopsychology, 8(4), 228238. https://doi.org/10.1089/eco.2016.0017.

Sociedad Mexicana de Psicología (2009). Código ético del psicólogo. Ciudad de México, México: Trillas.

Thompson, B. (2004). Exploratory and confirmatory factor analysis understanding Concepts and Applications. Washington, DC. EUA: American Psychological Association.

Van de Vyver, J., \& Abrams, D. (2015). Testing the prosocial effectiveness of the prototypical moral emotions: Elevation increases benevolent behaviors and outrage increases justice behaviors. Journal of Experimental Social Psychology, 58, 23-33. https://doi.org/10.1016/j.jesp.2014.12.005.

Wang, M., Wang, J., Deng, X., \& Chen, W. (2019). Why are empathic children more liked by peers? The mediating roles of prosocial and aggressive behaviors. Personality and Individual Differences, 144, 19-23. https://doi.org/10.1016/j.paid.2019.02.029.

Warneken, F., \& Tomasello, M. (2009). Varieties of altruism in children and chimpanzees. Trends in Cognitive Sciences, 13(9), 397-402. https://doi.org/10.1016/j.tics.2009.06.008.

Wilson J. (2000). Volunteering. Annual Review of Sociology, 26, 215-40.

https://doi.org/10.1146/annurev.soc.26.1.215. 